\title{
HDAC6 inhibitor WT161 induces apoptosis in retinoblastoma cells and synergistically interacts with cisplatin
}

\author{
Jun Sun ${ }^{1,2 \#}$, Xia Qian ${ }^{2 \#}$, Feifei Zhang ${ }^{2}$, Xiaofeng Tang ${ }^{2}$, Cheng Ju ${ }^{2,3}$, Renfeng Liu ${ }^{2,3}$, Ruihao Zhou ${ }^{2}$, \\ Zhiping Zhang ${ }^{3}$, Xiao-Bin Lv $^{2}$, Changhua Zhang ${ }^{1}$, Guofu Huang $^{4}$ \\ ${ }^{1}$ College of Pharmacy, Jiangxi University of Traditional Chinese Medicine, Nanchang 330004, China; ${ }^{2}$ Nanchang Key Laboratory of Cancer \\ Pathogenesis and Translational Research, Center Laboratory, The Third Affiliated Hospital of Nanchang University, Nanchang 330008, China; \\ ${ }^{3}$ Nanchang Key Laboratory of Orthopedics, The Third Affiliated Hospital of Nanchang University, Nanchang 330008, China; ${ }^{4}$ Department of \\ Ophthalmology, The Third Affiliated Hospital of Nanchang University, Nanchang 330008, China \\ Contributions: (I) Conception and design: C Zhang, G Huang; (II) Administrative support: Z Zhang, XB Lv; (III) Provision of study materials or \\ patients: C Ju, R Liu, R Zhou; (IV) Collection and assembly of data: F Zhang, X Tang; (V) Data analysis and interpretation: J Sun, X Qian; (VI) \\ Manuscript writing: All authors; (VII) Final approval of manuscript: All authors. \\ \#These authors contributed equally to this work. \\ Correspondence to: Changhua Zhang. College of Pharmacy, Jiangxi University of Traditional Chinese Medicine, Nanchang 330004, China. \\ Email: 20070964@jxutcm.edu.cn; Guofu Huang. Department of Ophthalmology, The Third Affiliated Hospital of Nanchang University, Nanchang \\ 330008, China. Email: hgf2222@sina.com.
}

\begin{abstract}
Background: WT161 is a recently discovered histone deacetylase 6 (HDAC6) inhibitor which shows anti-tumor effects on multiple myelomas and breast cancer. However, the role of WT161 in retinoblastoma remains unclear. The aim of this study is to explore the role of WT161 in retinoblastoma and its underlying mechanisms.
\end{abstract}

Methods: The anti-proliferation of WT161 on retinoblastoma cells was examined using 3-(4,5-dimethylthiazol-2-yl)-2,5-diphenyltetrazolium bromide (MTT) assay and soft agar colony formation assay. Cell apoptosis was analyzed using flow cytometer. WT161 and DDP synergistic effect was evaluated by isobologram analysis using CompuSyn software.

Results: WT161 suppressed the cell growth and induced apoptosis of retinoblastoma cells in a dose- and time-dependent manner. Mechanistically, WT161 increases the transcription of Bad through activating Bad promoter. Chromatin immunoprecipitation (ChIP) assay showed WT161 treatment increased acetylated histone $\mathrm{H} 3(\mathrm{AcH} 3)$ and acetylated histone $\mathrm{H} 4(\mathrm{AcH} 4)$ on the Bad promoter in retinoblastoma cells. In addition, WT161 shows synergistically inhibitory effects on retinoblastoma cell combined with cisplatin.

Conclusions: These results indicate that WT161, as a selective HDAC6 inhibitor, is a promising agent against retinoblastoma.

Keywords: WT161; retinoblastoma; apoptosis; Bad; synergistic inhibition; cisplatin

Submitted Jun 11, 2019. Accepted for publication Oct 10, 2019.

doi: $10.21037 /$ tcr.2019.10.30

View this article at: http://dx.doi.org/10.21037/tcr.2019.10.30

\section{Introduction}

Retinoblastoma is the most universal primary intraocular malignancy in childhood, accounting for $3 \%$ of childhood cancers $(1,2)$. Retinoblastoma can cause serious damage to the patient's vision and even lead to blindness. In addition, if left untreated, it can cause life-threatening problems due to local or distant metastasis (3). Chemotherapy is still a major means of treating retinoblastoma to date. However, many patients with retinoblastoma develop resistance and are at risk of blindness during the chemotherapy period $(4,5)$. Thus, it is necessary for us to discover novel anticancer agents that can reduce the drug resistance and toxicity during treatment. 
Genetic mutations are the leading cause of cancer development and progression. In addition, epigenetic abnormalities play a crucial role in the development of tumors (6). Therefore, epigenetic regulation has become a new treatment strategy for cancer. Epigenetic regulations of gene expression include DNA methylation, histone modification and Chromatin remodeling with reversible properties (7). Eleven human histone deacetylase (HDAC) family members have been identified and divided into four classes until now (8). They play a key role in epigenetic regulations of gene expression by regulating the acetylation level of the histone proteins (9). And an increasing number of research have demonstrated an anti-tumor role of HDAC inhibitors in multiple malignant tumors (10-12). Among the HDAC family, HDAC6 is a unique HDAC that not only deacetylates histones but also affects some nonhistone substrates such as heat shock protein 90 (HSP90) and $\alpha$-tubulin (13). Selective inhibition of HDAC6 plays anti-tumor effects through induction of differentiation, apoptosis, chemosensitivity, cell cycle arrest, and inhibition of migration and angiogenesis (14). More importantly, HDAC6 knockout mice develop normally and have seemingly normal major organ functions, suggesting that HDAC6 inhibition could not cause severe adverse events compared to inhibition of other HDACs (15). In addition, it has been found that the depletion of HDAC6 can enhance cisplatin-induced DNA damage (16). This suggests that inhibition of HDAC6 may enhance the sensitivity of chemotherapy by cisplatin. The selective HDAC6 inhibitors have been shown to have antitumor effects in a variety of tumors, such as multiple myeloma, melanoma, colorectal cancer, Gastric cancer and lymphoma (9,17-20). However, the role of selective HDAC6 inhibitors in retinoblastoma is rarely reported.

WT161 is a recently discovered potent and selective HDAC6 inhibitor. Recently, WT161 has been shown to play anti-tumor effects on multiple myeloma and breast cancer. In addition, WT161 shows a significantly synergistic antitumor effect with Bortezomib and this combination treatment is effective in bortezomib-resistant cells in multiple myeloma $(21,22)$. However, the role of WT161 in retinoblastoma remains unclear. Thus, the aim of this study is to explore the role of WT161 in retinoblastoma and its potential mechanisms.

\section{Methods}

\section{Cell culture and chemicals}

The human retinoblastoma cancer cell lines Y79 and Weri-
Rb1 were obtained from the Institute of Biochemistry and Cell Biology of the Chinese Academy of Sciences (Shanghai, China). Y79 cell was cultured in DMEM (Gibco, Thermo Fisher Scientific, Waltham, MA, USA) medium supplied with $10 \%$ fetal bovine serum (FBS) (Gibco-Life Technologies) at $37{ }^{\circ} \mathrm{C}$ in $5 \% \mathrm{CO}_{2}$ atmosphere. WT161 was purchased from MedChemExpress (NJ 08852, USA) and prepared in dimethyl sulfoxide (DMSO) (Sigma-Aldrich, St. Louis, MO, USA).

\section{3-(4,5-dimethylthiazol-2-yl)-2,5-diphenyltetrazolium bromide (MTT) assay}

Retinoblastoma cell was seed in 96-well plates at a density of 5,000 cells/well. The cells were treated with a series concentration of WT161 and/or cisplatin for 24, 48, and 72 h. MTT (Biosharp, China) was added to each well and cultured for $4 \mathrm{~h}$. Then the cells were lysed with DMSO and the optical density (OD) value at $490 \mathrm{~nm}$ was measured by microplate reader. The relative OD value $=\mathrm{OD}$ value of experimental well/OD value of control well.

\section{Soft agar colony formation assay}

We prepared $1.2 \%$ and $0.7 \%$ agarose (A9045-5G, Sigma), DMEM containing $20 \%$ FBS and $2 \times$ working concentration of penicillin streptomycin. The $1.2 \%$ agarose was diluted into the culture medium at a ratio of $1: 1$ and put into 6-wellplate with $2 \mathrm{~mL} /$ well to make bottom layer. To make upper layer of agarose, we diluted $0.7 \%$ agarose in the medium containing $1 \times 10^{3}$ single cell at a ratio of $1: 1$. The upper layer of agarose was added to 6-well-plate with $2 \mathrm{~mL} /$ well. After the upper layer of agar became solidified, each well was treated with different concentrations of WT161 for 2 weeks. After 2 weeks, the cells were stained with $0.1 \%$ crystal violet dye overnight. Colonies containing $>50$ cells were photographed and counted.

\section{Apoptosis assay}

We used the annexin V-FITC/propidium iodide (PI) apoptosis detection kit (BD Biosciences, USA) to assess the apoptosis of retinoblastoma cells. Cells were plated in 6-well plate and treated with specific concentration range of WT161 for $48 \mathrm{~h}$. Then the cells were harvested and washed twice with phosphate-buffered saline (PBS). At last, the cells were stained with annexin V-FITC and PI and the apoptosis rate was determined by flow cytometer (BD Biosciences). 


\section{Real-time polymerase chain reaction (RT-PCR)}

RT-PCR was performed as described previously (23). Primer sequences were as follows: human Bad forward: GACCAGCAGCCCAGAGTAT and reverse: CGCCTCCATGATGACTGTTATTG; human Bax forward: GAGGCAGCGGCAGTGATG and reverse: TCCTGGATGAAACCCTGTAGCA; human Bim forward: CGACAGTCTCAGGAGGAACC and reverse: CCTTCTCCATACCAGACGGA; human Noxa forward: ATGAATGCACCTTCACATTCCTCT and reverse: TCCAGCAGAGCTGGAAGTCGAGTGT; $\beta$-actin was used as an internal control and the primers were forward: CACTGTGTTGGCGTACAGGT, reverse: TCATCACCATTGGCAATGA.

\section{Luciferase reporter assay}

The Bad promoter was constructed into pMIR-reporter plasmids. The Bad and negative control (NC) mimics were transfected with Lipofectamine 2000 (Invitrogen, USA), respectively. Then 293T cells were cultured for 48 hours with different concentrations of WT161. The luciferase activities were detected by the luciferase reporter assay system.

\section{Western blot analysis}

The cells were washed 1-2 times using PBS and then added radioimmunoprecipitation assay (RIPA) buffer with protease inhibitors incubating on ice for $30 \mathrm{~min}$. The lysates were collected and centrifuged at $1,000 \mathrm{rpm}$ for $20 \mathrm{~min}$ at $4{ }^{\circ} \mathrm{C}$. We used the bicinchoninic acid (BCA) assay kit (Pierce, Rockford, IL, USA) to measure protein concentration. The proteins of each group were fractionated by SDSPAGE, and then transferred to PVDF membrane. After that, the membranes were soaked in PBST with $5 \%$ nonfat dry milk for 1.5 hours at room temperature and then probed with primary antibodies overnight at $4{ }^{\circ} \mathrm{C}$. After wash extensively, the membranes were probed with second antibody and then wash with PBST for 3 times. Then the proteins were visualized by chemiluminescence (Beyotime Biotechnology, Shanghai, China).

\section{Chromatin immunoprecipitation (ChIP) assay}

ChIP assays were used to examine the levels of acetylated histone $\mathrm{H} 3(\mathrm{AcH} 3)$ and acetylated histone
$\mathrm{H} 4$ (AcH4) on the Bad promoter region according to the instructions of ChIP assay kit (Millipore, USA). Chromatin was immunoprecipitated with antibodies against control IgG, acetyl-H3 and acetyl-H4. RT-PCR was performed for the Bad promoter region. Primer sequences were as follows: ChIP Bad proximal forward: TCCTTTCCTTATCTCCAGTGCC and reverse: GTGATTATTGTTGCTATTGTTACCG. The relative DNA levels in each immunoprecipitated group were normalized to the input DNA.

\section{Evaluation of drug interaction}

Evaluation of drug interaction was conducted as described previously (24). Briefly, using drug concentrations based on the half maximal inhibitory concentration $\left(\mathrm{IC}_{50}\right)$ value of each drug as a single drug to produce growth inhibition of about $10 \%$ to $90 \%$. When the drugs are used in combination, the drug concentration of the two drugs should be kept at a certain ratio. The combination index (CI) was calculated by the CompuSyn software (Biosoft).

\section{Statistical analysis}

We used statistical software SPSS 16 to analyze the experimental data. Results are shown as the mean \pm standard deviation (SD). We used LSD test to detect the statistical difference between experimental groups. $\mathrm{P}$ values less than 0.05 were considered statistically significant.

\section{Results}

\section{WT161 suppresses the cell growth of retinoblastoma cells}

Previous study had demonstrated that WT161 inhibits the growth of the breast cancer cells (21). To investigate whether WT161 exerts an anti-tumor effect on retinoblastoma, Y79 and Weri-Rb1 cells were treated with a series concentration of WT161 for indicated time. The enhancement of acetyl- $\alpha$-tubulin level by WT161 treatment in Y79 cells indicated the efficiency of WT161 in retinoblastoma cells (Figure 1A). MTT assay was used to detect the cell viability. As shown in Figure $1 B, C$, WT161 suppressed the cell growth of retinoblastoma cell in a dose- and time-dependent manner. In addition, to further investigate the effect of WT161 on suspension cell Y79, we performed the soft agar colony forming assay (25). As shown in Figure 1D, the results revealed that WT161 
A

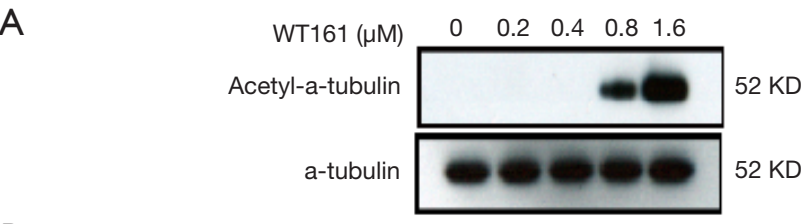

B
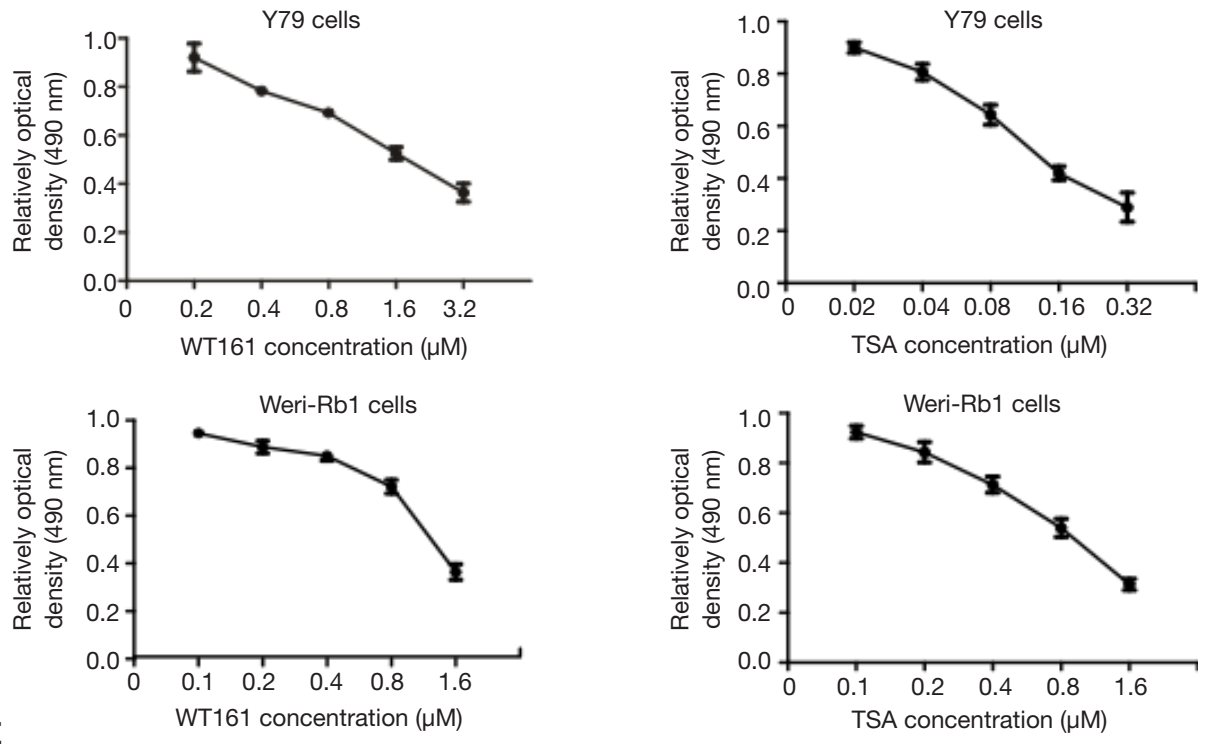

C
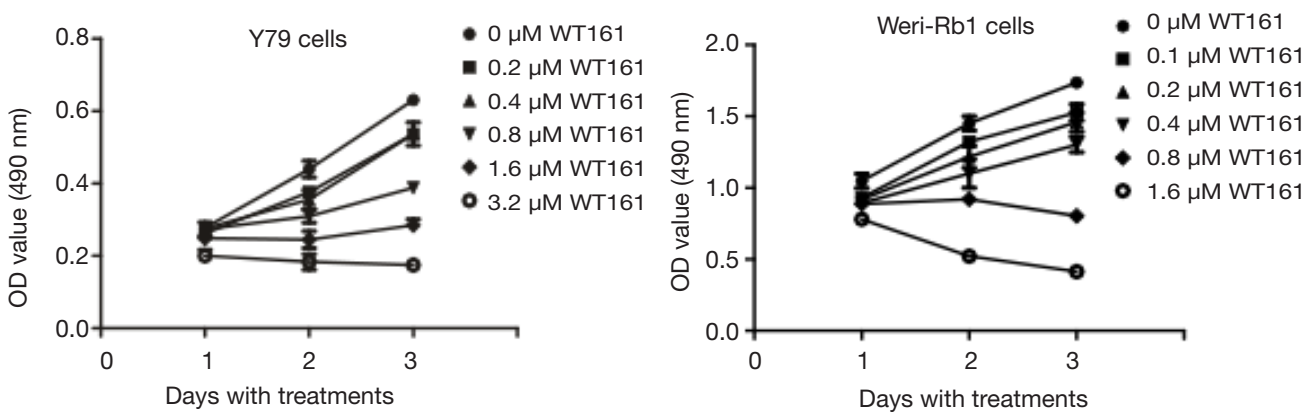

D

WT161
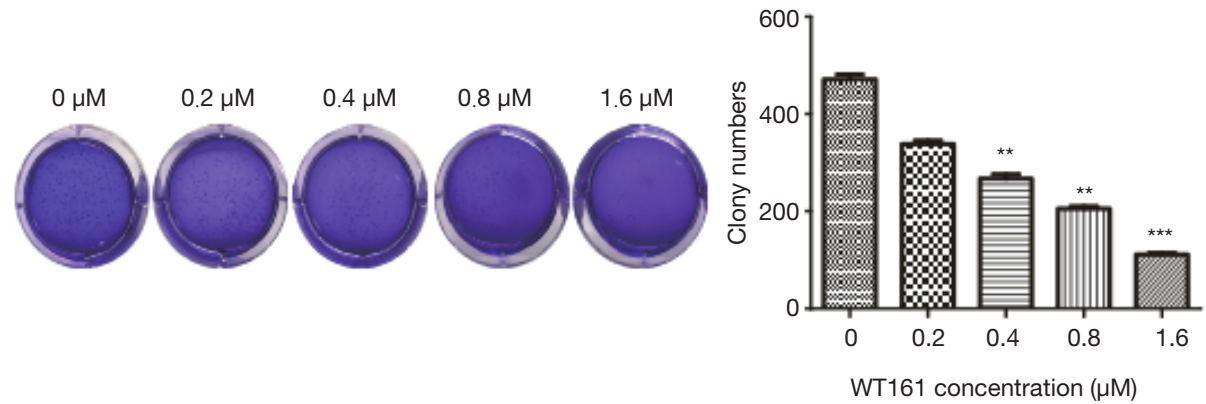

Figure 1 WT161 suppresses the cell growth of retinoblastoma cell line in dose- and time-dependent manners. (A) The protein expression of acetylated tubulin was determined by western-blotting analysis; (B) retinoblastoma cells were subjected to various concentrations of WT161 for $48 \mathrm{~h}$. TSA was as a positive control. Means from three independent experiments are presented as data; (C) various concentrations of WT161 was administered to retinoblastoma cells for $72 \mathrm{~h}$ with quantitative absorbance per $24 \mathrm{~h}$; (D) retinoblastoma cells were inoculated into 6-well plates with soft agar (1,000 cells/well). Each well was treated with different concentrations of WT161 for 2 weeks. After two weeks, the cells were stained $0.1 \%$ crystal violet dye overnight. $\mathrm{n}=3$, ${ }^{* *} \mathrm{P}<0.01,{ }^{* * *} \mathrm{P}<0.001$, compared with $\mathrm{NC} /$ vector. TSA, trichostatin $\mathrm{A}$; NC, negative control. 
inhibits the colony formation of Y79 cells in a dosedependent manner.

\section{WT161 treatment induces the apoptosis of retinoblastoma cells}

In order to determine whether WT161 affect the apoptosis of retinoblastoma cells, we tested apoptotic cell number of retinoblastoma cell through flow cytometry. The retinoblastoma cell line was cultured with a series concentration of WT161 for 48 hours. As shown in Figure 2A, WT161 induced a dose-dependent increase in the apoptotic cells. In addition, western blotting showed that caspase- 3 and PARP were activated upon WT161 treatment (Figure 2B). These results indicate that WT161 increases the apoptosis of retinoblastoma cell in a dosedependent manner.

\section{WT161 increases the apoptosis of retinoblastoma cells mainly through increasing the expression of Bad}

The BH3-only proteins of the BCL-2 family are important promoters of apoptosis, which transmits both extrinsic and intrinsic cell death signals (26). Thus, we want to explore whether WT161 induced the apoptosis of retinoblastoma cells through the $\mathrm{BH} 3$-only proteins. As shown in Figure $3 \mathrm{~A}$, the result showed that WT161 increased the mRNA expression level of several $\mathrm{BH} 3$-only proteins to varying degrees. Among them, Bad was the most pronouncedly upregulated gene induced by WT161. Besides, analyzing the high-through transcriptional factor ChIP-seq data through UCSC website, we found there are interaction of p300 and SIN3A-HDAC with the Bad promoter region, indicating Bad promoter might be regulated by histone acetylation. More important, the histone modification by ChIP-seq data from UCSC website showed heavily acetylation histone in Bad promoter. We hence examined whether WT161 affected the transcription of Bad through modulating promoter activity. Indeed, treatment of Y79 cells with WT161 increased the transcriptional level of Bad (Figure 3B). AcH3 and $\mathrm{AcH} 4$ are important substrates of HDAC6. We hence investigated whether WT161 influenced the expression of Bad by affecting the acetylation level of histone $\mathrm{H} 3$ and $\mathrm{H} 4$ on the $\mathrm{Bad}$ promoter as a HDAC6 inhibitor. As expected, WT161 treatment obviously increased the acetylation level of histone $\mathrm{H} 3$ and $\mathrm{H} 4$ on the Bad promoter (Figure 3C). Altogether, these results show that WT161 increased the transcription level of the Bad by increasing the acetylation level of histone $\mathrm{H} 3$ and $\mathrm{H} 4$ on the promoter.

\section{WT161 shows synergistic effects on retinoblastoma cell combined with cisplatin}

Cisplatin is a conventional chemotherapy drug for retinoblastoma treatment (27). Thus, we explored whether WT161 could show synergistic inhibitory effects on retinoblastoma cells combined with cisplatin. As shown in Figure 4, the results showed the majority of the CI values are lower than 0.7 , indicating that WT161 enhanced the inhibitory effect of cisplatin on retinoblastoma cells.

\section{Discussion}

Retinoblastoma is the most universal primary intraocular malignant tumor occurring in childhood. And it accounts for $3 \%$ all of childhood cancers. If not treated in time, it will be deadly. In recent years, the survival rate of patients with retinoblastoma has increased significantly due to the development of early cancer treatment. However, many patients with retinoblastoma are at risk of blindness due to metastasis or resistant-chemotherapy. It is critical for us to explore new anticancer agents for retinoblastoma.

In recent years, growing evidence indicates that epigenetic processes play an important role in tumorigenesis and progression. HDAC inhibitors play an important role in epigenetic regulation through modulating acetylation level of histones. Thus, HDAC inhibitors have become a new treatment strategy for cancer $(28,29)$. Previous studies have shown that WT161 exerted anti-tumor effects on multiple myeloma and breast cancer. A recently identified potent and selective HDAC6 inhibitor with an $\mathrm{IC}_{50}$ value of $0.40 \mathrm{nM}(21,22)$. In this study, we demonstrated that WT161 suppressed the cell growth and increases the apoptosis of retinoblastoma cells through increasing the acetylated $\mathrm{H} 3$ and $\mathrm{H} 4$ in the Bad promoter to regulate $\mathrm{Bad}$ expression. And it also showed synergistic inhibitory effects with cisplatin. Our results indicate that WT161 might become a new therapeutic approach for clinical treatment of retinoblastoma in the future.

Apoptosisis a process of programmed cell death regulated at the genetic level that results in the orderly and efficient removal of damaged cells (30). The dysregulation of apoptosis program is bound up with cell proliferation, the development of cancer and the resistance of tumors to drugs $(31,32)$. For this reason, the dysregulation of apoptosis 

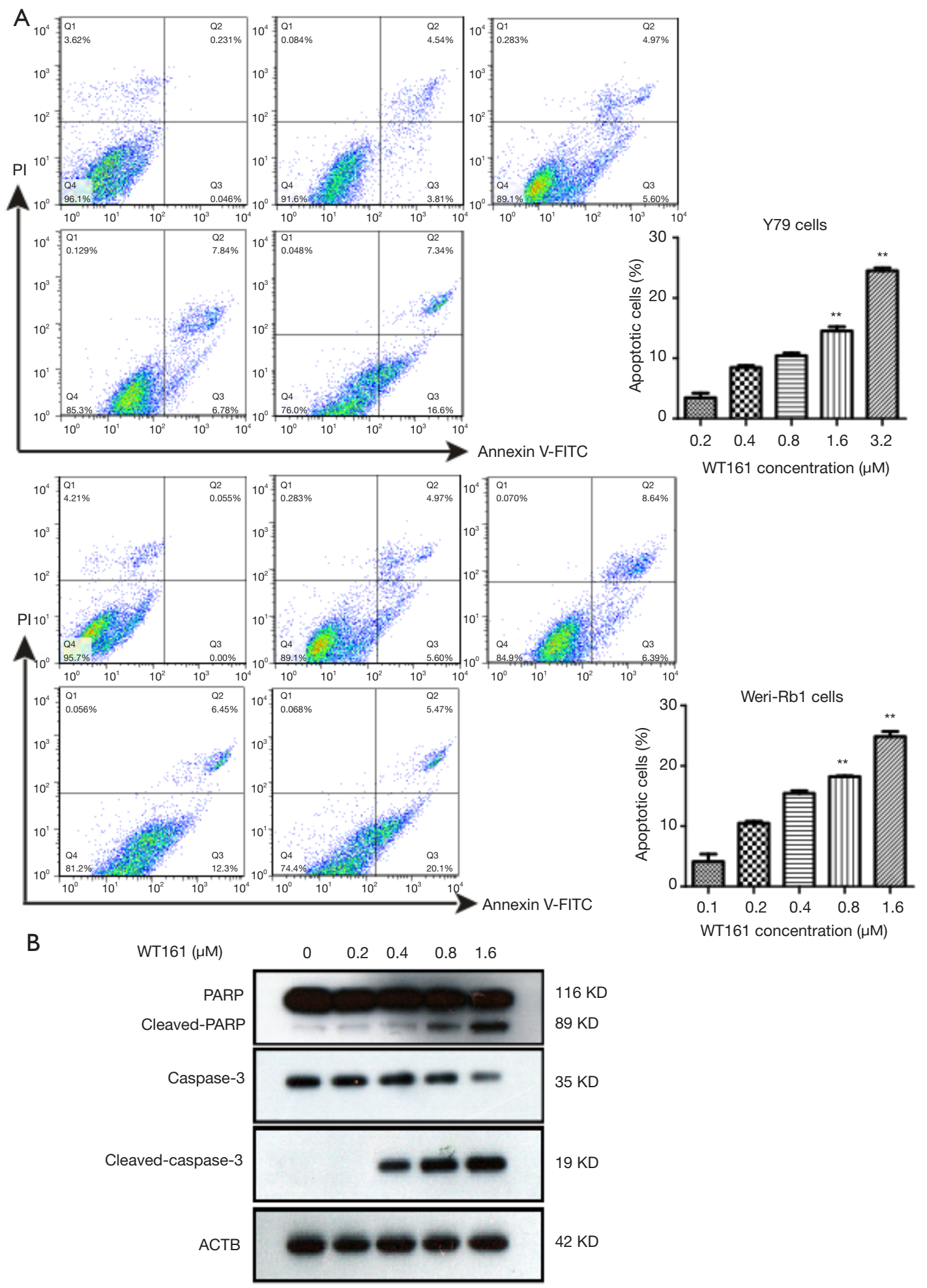

Figure 2 WT161 increases the apoptosis of retinoblastoma cell in a dose-dependent manner. (A) Retinoblastoma cells were subjected to various concentrations of WT161 for $48 \mathrm{~h}$. The cell apoptosis levels were assessed by flow cytometry; (B) the protein expression of caspase-3, cleaved caspase-3, PARP were determined by western-blotting analysis. $\mathrm{n}=3,{ }^{* *} \mathrm{P}<0.01$ compared with NC/vector. NC, negative control. 


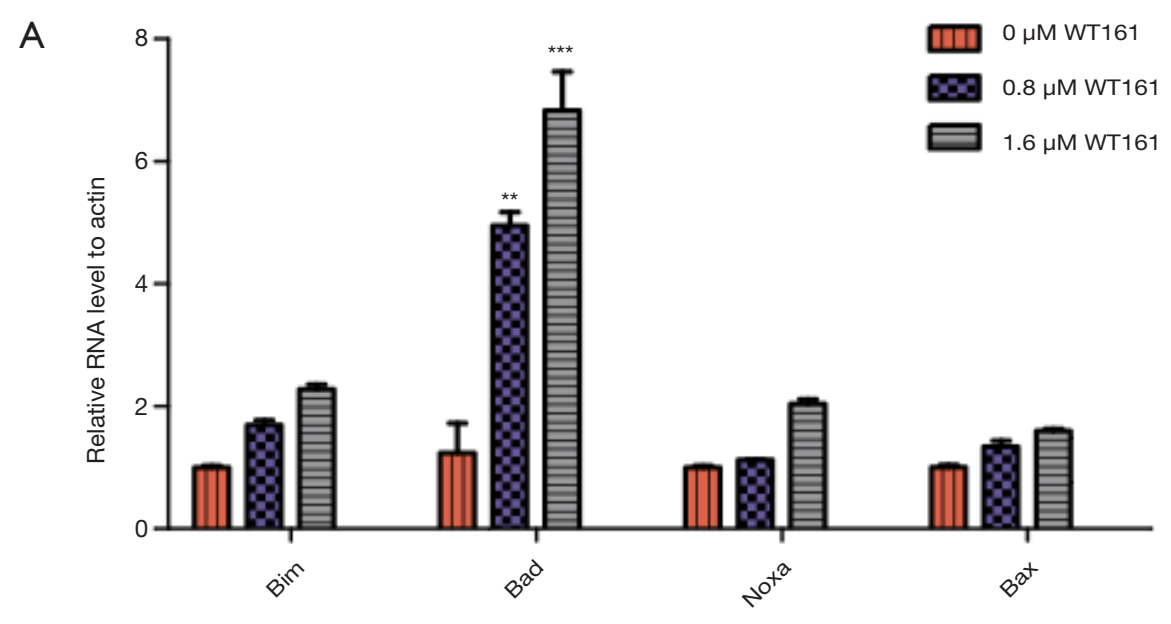

B

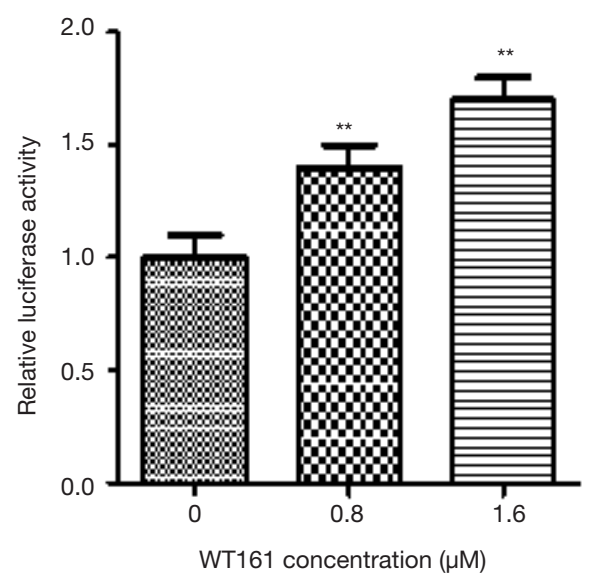

C
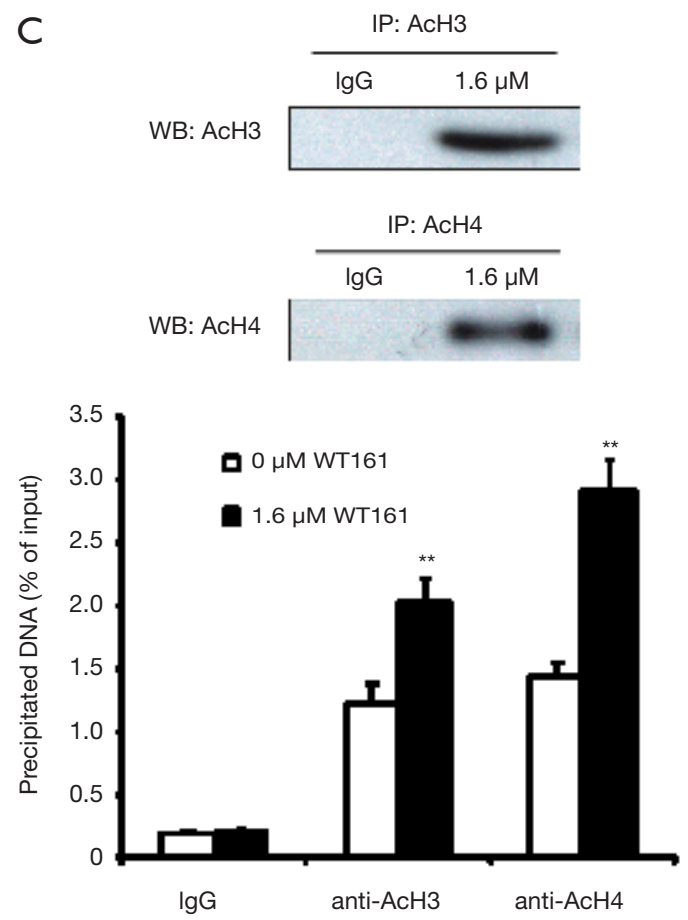

Figure 3 WT161 induced the apoptosis of retinoblastoma cell through increasing the expression of Bad. (A) The mRNA expression levels of Bim, Bad, Noxa and Bax were tested in cells treated with (0-1.6 $\mu \mathrm{M})$ WT161 for $48 \mathrm{~h}$ using the RT-PCR analysis; (B) the cells transfected with Bad or NC mimics were treated with 0.8 or $1.6 \mu \mathrm{M}$ WT161 for $48 \mathrm{~h}$. Then the luciferase activity was detected by luciferase reporter assay; (C) retinoblastoma cells were treated with $1.6 \mu \mathrm{M}$ WT161 for 48 h. The ChIP assay kit was used to check the level of AcH3 and AcH4 on the Bad promoter region. $\mathrm{n}=3,{ }^{* *} \mathrm{P}<0.01,{ }^{* * *} \mathrm{P}<0.001$. RT-PCR, real-time polymerase chain reaction; NC, negative control; ChIP, chromatin immunoprecipitation; AcH3, acetylated histone H3; AcH4, acetylated histone H4; IP, immunoprecipitation; WB, Western blot.

is considered to be one of the hallmarks of cancer (33). Therefore, the drugs that can induce apoptosis are a promising approach for the cancer treatment. As shown in Figure 2, our results showed that WT161 increased the apoptosis of retinoblastoma cell in a dose-dependent manner.

The pro-survival and pro-apoptotic members of BCL-2 protein family control the mitochondrial apoptotic pathway. 


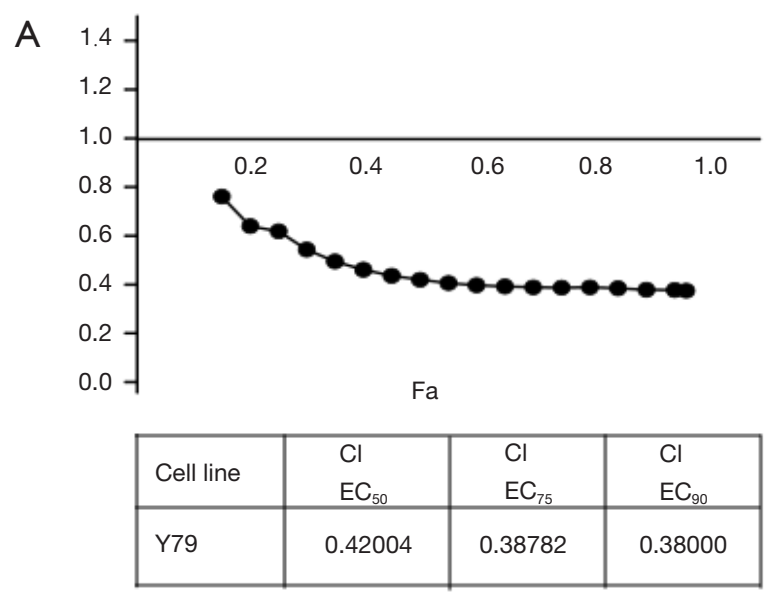

B

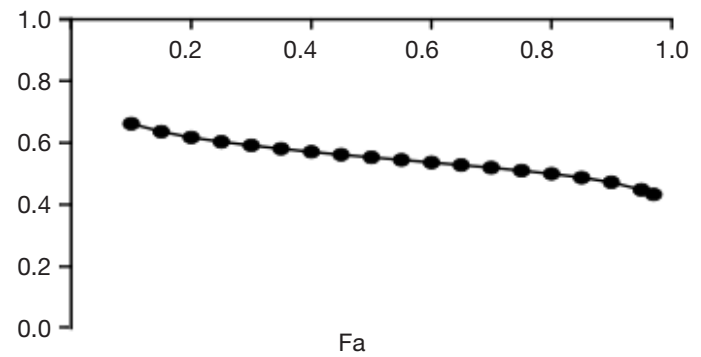

\begin{tabular}{|l|c|c|c|}
\hline Cell line & $\begin{array}{c}\mathrm{Cl} \\
\mathrm{EC}_{50}\end{array}$ & $\begin{array}{c}\mathrm{Cl} \\
\mathrm{EC}_{75}\end{array}$ & $\begin{array}{c}\mathrm{Cl} \\
\mathrm{EC}_{90}\end{array}$ \\
\hline Weri-Rb1 & 0.55388 & 0.51063 & 0.47257 \\
\hline
\end{tabular}

Figure 4 WT161 shows synergistic inhibitory effects on retinoblastoma cell combined with cisplatin. (A,B) CI-Fa graphs of combination studies with WT161 and cisplatin in the retinoblastoma cell lines Y79 and Weri-Rb1. When CI $<1$, it represents synergism; conversely, $\mathrm{CI}>1$ indicates antagonism. $\mathrm{CI}=1$ reveals additivity. And $\mathrm{Fa}=$ fraction was affected by the combination treatment. CI, combination index; EC, effective concentration.

The balance of them can regulate programmed cell death appropriately. When pro-apoptotic proteins predominate, they will induce the cell apoptosis $(34,35)$. In our study, we discovered that WT161 increased the mRNA expression level of several pro-apoptotic members to varying degrees. And Bad was the most pronouncedly influenced by WT161. In recent years, great progress has been obtained in understanding how histone acetylation and deacetylation influence promoter activity. And histone acetylation has become a key regulatory mechanism for controlling multiple nuclear processes and transcription (36). In addition, a previous study indicated that suppression of HDAC6 increased Pax7 expression through increasing the acetylation of histones $\mathrm{H} 3$ and $\mathrm{H} 4$ on the $\mathrm{Pax} 7$ promoter (37). In this study, we demonstrated that WT161, as a HDAC6 inhibitor, increased the transcription of Bad by affecting the $\mathrm{AcH} 3$ and $\mathrm{AcH} 4$ on the Bad promoter.

As a well-known chemotherapy drug, cisplatin has been certificated to combat different types of cancer including sarcoma, soft tissue cancer, bones, muscles and blood vessels (38). In retinoblastoma, cisplatin is also used as a common clinical chemotherapy regimen (39). However, clinical use of cisplatin usually induces drug resistance, ototoxic and nephrotoxic $(40,41)$. Thence, the combination of cisplatin with other pharmaceutical agents may improve its efficacy and reduce the toxicity on patients. In the study, our results indicated that WT161 enhanced the cytotoxicity of DDP and showed a significantly synergistic effect when combined with DDP, suggesting that the combined treatment of WT161 and cisplatin could be an effective strategy for the treatment of retinoblastoma.

In conclusion, we demonstrated the important role of WT161 in retinoblastoma. Our results showed that WT161 inhibits the cell growth and induces the apoptosis of retinoblastoma cells in a dose- and time-dependent manner. In addition, WT161 shows synergistic inhibitory effects on retinoblastoma cell combined with cisplatin. WT161 might be a promising agent against retinoblastoma.

\section{Acknowledgments}

Funding: This study was partially sponsored by grants from the National Natural Science Foundation of China (81560158 to GFH, 81774194 to CHZ); the Natural Science Key Project Joint Foundation of Jiangxi Province (20192ACBL20027 to CHZ); the Natural Science Foundation of Jiangxi Province (20171ACB21073 to XBL); Excellent Youth Foundation of Jiangxi Scientific Committee (No. 20162BCB23001 to XBL); The Foundation of Nanchang Science and Technology Bureau (No. 2016 ZSCX 009 to XBL).

\section{Footnote}

Conflicts of Interest: All authors have completed the ICMJE uniform disclosure form (available at http://dx.doi. org/10.21037/tcr.2019.10.30). The authors have no conflicts of interest to declare. 
Ethical Statement: The authors are accountable for all aspects of the work in ensuring that questions related to the accuracy or integrity of any part of the work are appropriately investigated and resolved.

Open Access Statement: This is an Open Access article distributed in accordance with the Creative Commons Attribution-NonCommercial-NoDerivs 4.0 International License (CC BY-NC-ND 4.0), which permits the noncommercial replication and distribution of the article with the strict proviso that no changes or edits are made and the original work is properly cited (including links to both the formal publication through the relevant DOI and the license). See: https://creativecommons.org/licenses/by-nc-nd/4.0/.

\section{References}

1. Dimaras H, Corson TW. Retinoblastoma, the visible CNS tumor: a review. J Neurosci Res 2019;97:29-44.

2. Rao R, Honavar SG. Retinoblastoma. Indian J Pediatr 2017;84:937-44.

3. Meel R, Radhakrishnan V, Bakhshi S. Current therapy and recent advances in the management of retinoblastoma. Indian J Med Paediatr Oncol 2012;33:80-8.

4. Fabian ID, Onadim Z, Karaa E, et al. The management of retinoblastoma. Oncogene 2018;37:1551-60.

5. Fabian ID, Puccinelli F, Gaillard MC, et al. Diagnosis and management of secondary epipapillary retinoblastoma. $\mathrm{Br}$ J Ophthalmol 2017;101:1412-8.

6. Schnekenburger M, Florean C, Dicato M, et al. Epigenetic alterations as a universal feature of cancer hallmarks and a promising target for personalized treatments. Curr Top Med Chem 2016;16:745-76.

7. Samadani AA, Norollahi SE, Rashidy-Pour A, et al. Cancer signaling pathways with a therapeutic approach: an overview in epigenetic regulations of cancer stem cells. Biomed Pharmacother 2018;108:590-9.

8. Sangwan R, Rajan R, Mandal PK. HDAC as onco target: reviewing the synthetic approaches with SAR study of their inhibitors. Eur J Med Chem 2018;158:620-706.

9. Bae J, Hideshima T, Tai YT, et al. Histone deacetylase (HDAC) inhibitor ACY241 enhances anti-tumor activities of antigen-specific central memory cytotoxic $\mathrm{T}$ lymphocytes against multiple myeloma and solid tumors. Leukemia 2018;32:1932-47.

10. Geng Y, Liu J, Xie Y, et al. Trichostatin A promotes GLI1 degradation and P21 expression in multiple myeloma cells. Cancer Manag Res 2018;10:2905-14.
11. Heers H, Stanislaw J, Harrelson J, et al. Valproic acid as an adjunctive therapeutic agent for the treatment of breast cancer. Eur J Pharmacol 2018;835:61-74.

12. Sanaei M, Kavoosi F, Roustazadeh A, et al. In vitro effect of the histone deacetylase inhibitor valproic acid on viability and apoptosis of the PLC/PRF5 human hepatocellular carcinoma cell line. Asian Pac J Cancer Prev 2018;19:2507-10.

13. Li T, Zhang C, Hassan S, et al. Histone deacetylase 6 in cancer. J Hematol Oncol 2018;11:111.

14. Li Y, Shin D, Kwon SH. Histone deacetylase 6 plays a role as a distinct regulator of diverse cellular processes. FEBS J 2013;280:775-93.

15. Zhang Y, Kwon S, Yamaguchi T, et al. Mice lacking histone deacetylase 6 have hyperacetylated tubulin but are viable and develop normally. Mol Cell Biol 2008;28:1688-701.

16. Wang L, Xiang S, Williams KA, et al. Depletion of HDAC6 enhances cisplatin-induced DNA damage and apoptosis in non-small cell lung cancer cells. PLoS One 2012;7:e44265.

17. Cosenza M, Civallero M, Marcheselli L, et al. Ricolinostat, a selective HDAC6 inhibitor, shows anti-lymphoma cell activity alone and in combination with bendamustine. Apoptosis 2017;22:827-40.

18. Dong J, Zheng N, Wang X, et al. A novel HDAC6 inhibitor exerts an anti-cancer effect by triggering cell cycle arrest and apoptosis in gastric cancer. Eur J Pharmacol 2018;828:67-79.

19. Wang F, Zhong BW, Zhao ZR. ACY 1215, a histone deacetylase 6 inhibitor, inhibits cancer cell growth in melanoma. J Biol Regul Homeost Agents 2018;32:851-8.

20. Won HR, Ryu HW, Shin DH, et al. A452, an HDAC6selective inhibitor, synergistically enhances the anticancer activity of chemotherapeutic agents in colorectal cancer cells. Mol Carcinog 2018;57:1383-95.

21. Hideshima T, Mazitschek R, Qi J, et al. HDAC6 inhibitor WT161 downregulates growth factor receptors in breast cancer. Oncotarget 2017;8:80109-23.

22. Hideshima T, Qi J, Paranal RM, et al. Discovery of selective small-molecule HDAC6 inhibitor for overcoming proteasome inhibitor resistance in multiple myeloma. Proc Natl Acad Sci U S A 2016;113:13162-7.

23. Ju C, Zhou R, Sun J, et al. LncRNA SNHG5 promotes the progression of osteosarcoma by sponging the miR212-3p/SGK3 axis. Cancer Cell Int 2018;18:141.

24. Su F, Bradley WD, Wang Q, et al. Resistance to selective BRAF inhibition can be mediated by modest upstream 
pathway activation. Cancer Res 2012;72:969-78.

25. Bich Loan NT, Trung NN, Le Na NT, et al. Anticancer activities of ricin-liposome complexes on SKMEL-28 cells. Asian Pac J Cancer Prev 2019;20:2117-23.

26. Shamas-Din A, Brahmbhatt H, Leber B, et al. BH3-only proteins: orchestrators of apoptosis. Biochim Biophys Acta 2011;1813:508-20.

27. Song HB, Jun HO, Kim JH, et al. Anti-apoptotic effect of clusterin on cisplatin-induced cell death of retinoblastoma cells. Oncol Rep 2013;30:2713-8.

28. De Souza C, Chatterji BP. HDAC inhibitors as novel anticancer therapeutics. Recent Pat Anticancer Drug Discov 2015;10:145-62.

29. Eckschlager T, Plch J, Stiborova M, et al. Histone deacetylase inhibitors as anticancer drugs. Int J Mol Sci 2017. doi: 10.3390/ijms18071414.

30. Fuchs Y, Steller H. Programmed cell death in animal development and disease. Cell 2011;147:742-58.

31. Fulda S. Tumor resistance to apoptosis. Int J Cancer 2009;124:511-5.

32. Plati J, Bucur O, Khosravi-Far R. Dysregulation of apoptotic signaling in cancer: molecular mechanisms and therapeutic opportunities. J Cell Biochem 2008;104:1124-49.

33. Hanahan D, Weinberg RA. Hallmarks of cancer: the next generation. Cell 2011;144:646-74.

Cite this article as: Sun J, Qian X, Zhang F, Tang X, Ju C, Liu R, Zhou R, Zhang Z, Lv XB, Zhang C, Huang G. HDAC6 inhibitor WT161 induces apoptosis in retinoblastoma cells and synergistically interacts with cisplatin. Transl Cancer Res 2019;8(8):2759-2768. doi: 10.21037/tcr.2019.10.30
34. Campbell KJ, Tait SWG. Targeting BCL-2 regulated apoptosis in cancer. Open Biol 2018. doi: 10.1098/ rsob. 180002.

35. Elkholi R, Floros KV, Chipuk JE. The Role of BH3-Only Proteins in Tumor Cell Development, Signaling, and Treatment. Genes Cancer 2011;2:523-37.

36. Forsberg EC, Bresnick EH. Histone acetylation beyond promoters: long-range acetylation patterns in the chromatin world. Bioessays 2001;23:820-30.

37. Lee SW, Yang J, Kim SY, et al. MicroRNA-26a induced by hypoxia targets HDAC6 in myogenic differentiation of embryonic stem cells. Nucleic Acids Res 2015;43:2057-73.

38. Dasari S, Tchounwou PB. Cisplatin in cancer therapy: molecular mechanisms of action. Eur J Pharmacol 2014;740:364-78.

39. Varan A, Kiratli H, Aydin B, et al. The treatment of retinoblastoma with four-drug regimen including cisplatin, etoposide, vincristine, and cyclophosphamide. Pediatr Hematol Oncol 2012;29:529-37.

40. Jia M, Wei Z, Liu P, et al. Silencing of ABCG2 by microRNA-3163 inhibits multidrug resistance in retinoblastoma cancer stem cells. J Korean Med Sci 2016;31:836-42.

41. Ruggiero A, Trombatore G, Triarico S, et al. Platinum compounds in children with cancer: toxicity and clinical management. Anticancer Drugs 2013;24:1007-19. 